\title{
Evaluation of Wearable Epileptic Seizure Monitors
}

\author{
Tendai Rukasha \\ Keele University \\ Keele, UK \\ t.rukasha@keele.ac.uk
}

\begin{abstract}
Wearable health devices that detect epileptic seizures have the potential to hail timely assistance for individuals, inform their treatment and assist care and self-management. New wearable seizuredetecting devices are becoming available to individuals, carers and researchers but there is scope for improvements in device performance and for more evaluations in the research literature. This position paper outlines research that includes a review of the evaluation literature and both quantitative and qualitative device evaluations.
\end{abstract}

Wearable devices, Health technology, Usability, Epilepsy, Seizure detection.

\section{INTRODUCTION}

Epilepsy is a neurological disorder that affects 50 million people worldwide. It is characterised by seizures that can present in very different ways, for example, from short absences to protracted convulsions. Although seizures can be controlled with antiepileptic drugs, $30 \%$ of people with epilepsy have drug-resistant seizures (Sheng et al., 2018). The onset of a seizure is associated with changes in temperature, perspiration and heart rate (Wannamaker et al.,1985; Baumgartner et al., 2001). These changes have the potential to be detected by wearable skin temperature, electrodermal activity (EDA) and optical pulse 'photoplethysmography' (PPG) sensors, respectively. During a seizure, rhythmic shaking movements or the lack of movement can be detected via signals from a wearable accelerometer. Wearable seizure-detecting devices that include these sensors are now becoming available to individuals, carers, healthcare professionals and researchers. However, reliable seizure detection is difficult in everyday life and devices can miss seizures and produce false alarms (Johansson et al., 2018).

\section{RESEARCH SCOPE}

The PhD research surveys and evaluates wearable epileptic seizure monitoring devices with the aim of contributing toward improving future device designs and evaluations.

\subsection{Research Directions}

The aims and objectives are as follows:

- To survey and evaluate wearable seizure monitor performance.

- To identify issues in wearable interface design and recommend improvements.

- To collect and analyse the opinions and experiences of people with epilepsy, carers, family, friends, alarm receivers and healthcare professionals.

The research questions that guide the research aims and objectives:

(i) How to evaluate the performance of wearable devices for epilepsy seizure monitoring?

(ii) To what extent do wearable user interface designs affect usability?

(iii) What are epilepsy stakeholders' perceptions and experiences of consumer wearable devices?

Systematic literature review. A systematic literature review of wearable seizure-monitoring device evaluations was performed and has been published in MDPI electronics journal (Rukasha et al., 2020). The devices and apps available are summarised in Figure 1. Published evaluations reported varying levels of detail about performance metrics such as sensitivity, specificity, positive predictive and negative predictive 
values, and false alarm rates for detecting different types of epileptic seizures.

The review demonstrated that despite a very large body of research into novel methods for seizure detection, there is a lack of research reporting evaluation data for available devices, and, in particular, there is a lack of studies reporting on realworld use and experiences of epilepsy stakeholders.

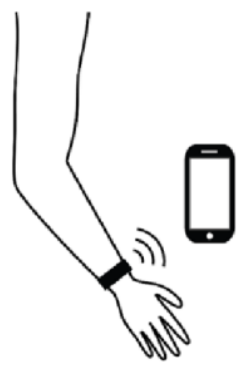

(a)

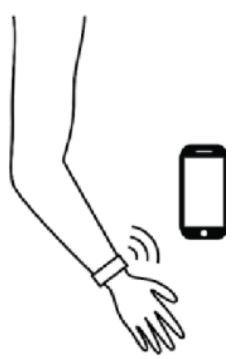

(b)

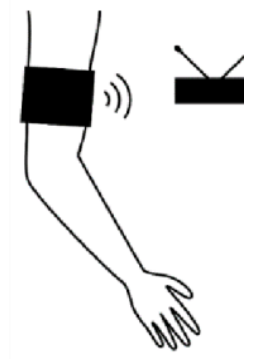

(c)
Figure 1. Wearables and apps for epilepsy seizure detection: (a) dedicated wrist-worn sensing device and companion app (e.g., Embrace (Empatica) and Epi-Care free (Danish Care Technology) devices); (b) app using sensed data from a compatible consumer wrist-worn tracker (e.g., the SmartWatch Inspyre app (Smart Monitor) with an Apple or Samsung device); (c) a nonwrist wearable with a base station (NightWatch (LivAssured, B.V) device).

Study One - Performance evaluation: Even in well-resourced clinical studies, it is still very challenging to test the performance of seizure detecting wearables because it requires the recruitment and observation of epileptic individuals into laboratory environments where EEG and/or other truth data can be achieved. But, seizures are intermittent and should not be provoked, so it may take very many hours of clinical resource to capture a sufficient number of seizures for device evaluation.

An alternative to seizure-monitoring evaluation is the evaluation of sensing performance. If wearable sensing devices are to perform well at detecting and monitoring seizures, they should perform well at recording their sensed values. However, reliable heart rate sensing is challenging during activity [Oniani, 2018].

In this study, heart rate sensing evaluations were completed for 12-hour everyday living and 15minute treadmill activity data collections. An Empatica E4 wrist-worn wearable (a research version of an Empatica Embrace epileptic seizure monitor) and the Polar ECG chest strap were used in testing. Figure 2 shows an example of the different recorded heart rates from both devices worn by the same participant during 12-hour everyday living

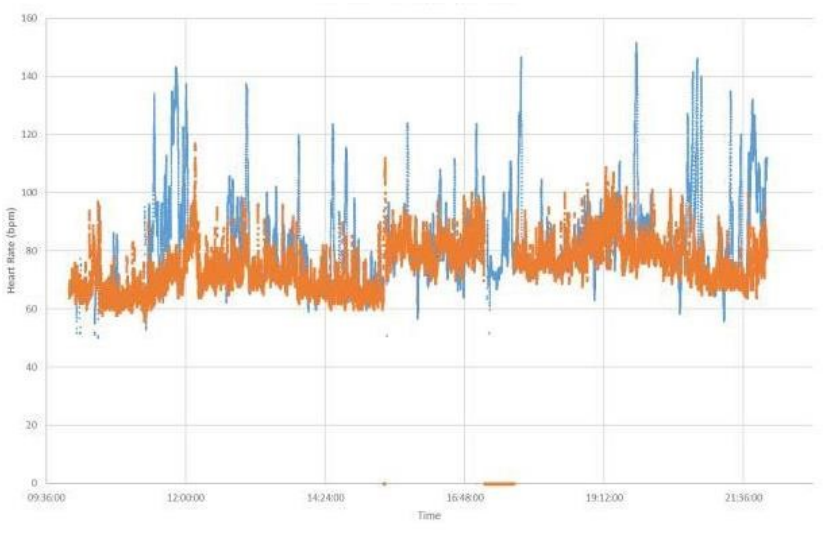

Figure 2. Example concurrent acquisitions of 12-hour everyday living heart rate recordings from a E4 wearable (blue line) and a Polar ECG chest strap (orange line).

Study Two - Interface Evaluation: This study involved a heuristic evaluation (using Neilsen's Usability Heuristics for User Interface Design [Neilsen, 1994]) of the minimal circular coloured light LED interface of the Empatica 'Embrace' wearable epilepsy seizure monitor.

Minimal interface indicators and alerts can quickly become familiar to individuals wearing devices every day. But, in critical healthcare applications there can be other 'stakeholder users' acting in support during episodes when the wearer may be incapacitated or confused [Rukasha, 2020b].

A number of participants assessors with $\mathrm{HCl}$ experience were recruited to perform a heuristic evaluation and to try to guess the meaning of different light patterns. Figure 3 shows box plot results for the light pattern indicating an "unusual event detected", i.e., reporting a potential seizure. As demonstrated by the example, assessors lacked confidence about the meaning of the display and had difficulties disambiguating between sets of possible conditions.

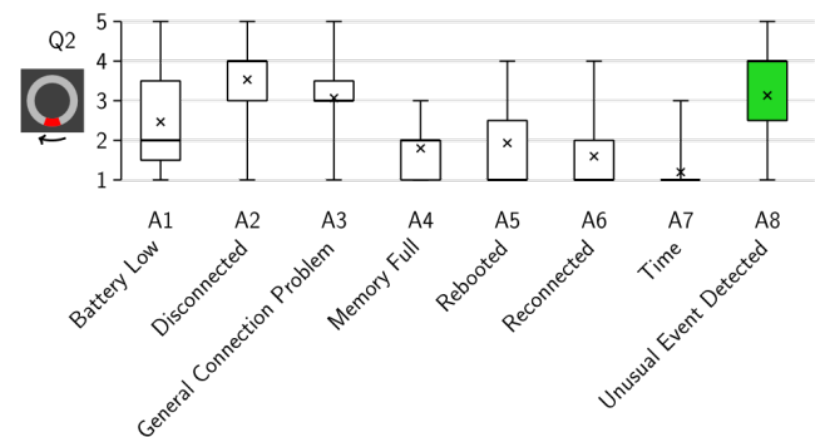

Figure 3. Box Plot Responses of Guessed Interface

$$
\begin{aligned}
& \text { Visualisation: Likert scale responses (5=Definitely is and } \\
& \text { 1= Definitely isn't): Q2: Displayed "Unusual event } \\
& \text { detected", the correct answer (A8) is shaded green. }
\end{aligned}
$$

Study Three - Stakeholder Evaluations: With Keele University ethical approval, a survey of stakeholder opinions and experiences collection is currently in 
progress. Three questionnaires survey 2 opinions and experiences for i) people with epilepsy, ii) carers, and iii) healthcare professionals. These questionnaires are available at the time of writing on the Epilepsy Action charity website,

https://www.epilepsy.org.uk/research/takepart/proje cts-you-can-take-part-in/wearabledevices. Epilepsy Action is a charity that improves the lives of people affected by epilepsy, by giving advice, improve healthcare and fund research and campaign for change.

\section{DISCUSSION}

The findings of the research so far indicate that there is enthusiasm for wearable epilepsy seizure monitoring among individuals, carers and health professionals but there are also concerns about performance and false alarm rates.

The review of the literature highlighted the lack of both qualitative and quantitative published research evaluating the devices. This research aims to contribute to the area.

\section{REFERENCES}

Baumgartner, C.; Lurger, S.; Leutmezer, F. Autonomic symptoms during epileptic seizures. Epileptic Disord. 2001, 3, 103-116.

Collins, T.; Woolley, S.I.; Oniani, S.; Pires, I.M.; Garcia, N.M.; Ledger, S.J.; Pandyan, A. Version Reporting and Assessment Approaches for New and Updated Activity and Heart Rate Monitors. Sensors 2019, 19, 1705.

Danish Care Technology (Epi-Care Free), Sorø,

Denmark, UK. Available online: https://danishcare.co.uk/epicare-free/ (accessed on 12 May 2020).

Empatica Inc. Boston, USA and Empatica Srl, Milano, Italy (E3, E4 and Embrace). Available online: https://www.empatica.com (accessed on 12 May 2020).
Jakob Nielsen. 1994. Enhancing the explanatory power of usability heuristics.In Proceedings of the SIGCHI conference on Human Factors in Computing Systems.ACM Press, 152-158. https://doi.org/10.1145/191666.191729

Johansson, D., Malmgren, K. And Alt Murphy, m. Wearable sensors for clinical applications in epilepsy, Parkinson's disease, and stroke: a mixedmethods systematic review. J. Neurology 2018., 265(8), 1740-1752.

LivAssured, B.V. (NightWatch), Leiden, The

Netherlands. Available online: https://www.nightwatch.nl/ (accessed on 12 May 2020).

Oniani, S., Woolley, S.I., Pires, I.M., Garcia, N.M., Collins, T., Ledger, S. and Pandyan, A., 2018. Reliability assessment of new and updated consumer-grade activity and heart rate monitors.

Rukasha, T., Woolley, S., Kyriacou, T. and Collins, T. Evaluation of Wearable Electronics for Epilepsy: A Systematic Review. Electronics, 2020, 9(6), p.968.

Rukasha, T.; Woolley, S. I.; Collins, T., Wearable Epilepsy Seizure Monitor User Interface Evaluation An Evaluation of the Empatica 'Embrace' Interface, ACM UbiComp 2020 (in press).

Sheng, J., Liu, S., Gin, H., Li, B. and Zhang, X., 2018. Drug-resistant epilepsy and surgery. Current neuropharmacology, 16(1), pp.17-28.

Smart Monitor (SmartWatch Inspyre), San Jose, USA. Available online: https://smartmonitor.com/aboutsmartwatchinspyreby-smartmonitor/ (accessed on 12 May 2020).

Wannamaker, B.B. Autonomic Nervous System and Epilepsy. Epilepsia 1985, 26, S31-S39

Woolley, S.; Collins, T.; Mitchell, J.; Fredericks, D. Investigation of wearable health tracker version updates. BMJ Health Care Inform. 2019, 26, e100083. 